\title{
GHRONOLOGY
}

\begin{abstract}
Tr
hat follows is an inventory of the more important monarchs, not a complete list. The Archaic Period is given more leeway here than in the text. Under Dynasties 18 and 19, I follow now an alternative resulting from recent proposals by M. L. Bierbrier, R. Krauss, and others, including fixing the beginning of the reign of Ramesses II at 1279 B.C.E., shortening the reign of Merneptah to ten years, and eliminating an independent reign for Amenmesse, though it cannot be regarded as definitive. The list is made up of the personal names of the kings, with the throne names added in parentheses from Dynasty 12 on; for Dynasty 11, the Horus names are added, and for Dynasty 1 , the Greek forms as handed down in the epitomes of Manetho.
\end{abstract}

\section{Dynasty 0}

Irihor (?)

"Scorpion"

C. 305 O B.C.E.

Narmer

\section{Archaic Period}

\section{Dynasty I}

c. $3000-2800$

Aha (Athothis)

Djer (Kenkenes) 
Wadj (Uenephes)

Den (Usaphais)

Adjib (Miebis)

Semerkhet (Semempses)

Qaa (Ubienthes)

\section{Dynasty 2}

C. $2800-2670$

Hetepsekhemwy

Reneb

Ninetjer

Peribsen

Khasekhem(wy)

\section{Old Kingdom}

\section{Dynasty 3}

C. $2670-2600$

Nebka

Djoser

Sekhemkhet

Huni

$2654-2635$

$2625-2600$

\section{Dynasty 4}

c. 2600-2487

Snofru

Khufu (Cheops)

Radjedef

Khephren (Khafre)

Menkaure (Mycerinos)

Shepseskaf

$$
\begin{aligned}
& 2600-2571 \\
& 2571-2548 \\
& 2548-2540 \\
& 2540-2514 \\
& 2510-2491 \\
& 2491-2487
\end{aligned}
$$

\section{Dynasty 5}

c. 2487-2348

Userkaf

Sahure

Neferirkare

Neuserre

Menkauhor

Djedkare (Izezi)

Wenis

$$
\begin{aligned}
& 2487-2480 \\
& 2480-2468 \\
& 2468-2449 \\
& 2443-2419 \\
& 2419-2411 \\
& 2411-2378 \\
& 2378-2348
\end{aligned}
$$

\section{Dynasty 6}

C. 2348-2198

Teti

2348-2320

Userkare 
Pepy I (Meryre)

2316-2284

Merenre I

2284-2270

Pepy II (Neferkare)

2270-2205

Merenre II. Antyemzaf

Nitocris (?)

\section{First Intermediate Period}

\section{Dynasties 7-8}

c. $2198-2160$

Several ephemeral rulers

\section{Dynasties 9-10}

c. 2160-1980

Kheti III

Merykare

\section{Dynasty ||}

c. $2081-1938$

Inyotef I (Sehertawy)

2081-2065

Inyotef II (Wahankh)

2065-2016

Inyotef III (Nakhtnebtepnufer)

2016-2008

Mentuhotpe I (Nebhepetre)

2008-1957

Mentuhotpe II (Sankhkare)

1957-1945

Mentuhotpe III (Nebtawyre)

1945-1938

\section{Middle Kingdom}

\section{Dynasty 12}

c. 1938-1759

(Overlapping dates are due to coregencies)

Amenemhet I (Sehetepibre)

Senwosret I (Kheperkare)

1938-1909

1919-1875

Amenemhet II (Nubkaure)

$1877-1843$

Senwosret II (Khakheperre)

$1845-1837$

Senwosret III (Khakaure)

$1837-1818$

Amenemhet III (Nimaatre)

1818-1773

Amenemhet IV (Maakherure)

$1773-1763$

Nefrusobk (Nefru Sobek Shedty)

$1763-1759$

\section{Second Intermediate Period}

\section{Dynasty 13}

c. $1759-1630$

(1) Wegaf (Khutawyre)

1759-1757

(12) Sebekhotpe I (Khaankhre) 
(14) Awibre

(16) Sebekhotpe II (Sekhemre-khutawy)

(17) Khendjer (Userkare)

(21) Sebekhotpe III (Sekhemre-swadjtawy)

(22) Neferhotep I (Khasekhemre)

1705-1694

(24) Sebekhotpe IV (Khaneferre)

1694-1685

(27) Aya (Merneferre)

and many others

\section{Dynasty 14}

c. $1700-1630$

Nehesy and other minor kings in the delta

Dynasties 15-16 (Hyksos)

c. $1630-1522$

Salitis (Sekhaenre?)

Bnon

Apachnan/Khiyan (Swoserenre)

Iannas/Yinassi

Arkhles/Sikruhaddu

Apophis (Awoserre)

1573-1533

Khamudi

1533-1522

Dynasty 17 (Theban)

c. $1640-1539$

Inyotef $\mathrm{V}$ (Nubkheperre)

c. 1630

Tao I (Senakhtenre)

Tao II (?) (Seqenenre)

c. 1570

Kamose (Wadjkheperre)

c. $1560 / 50$

1543-1539

\section{New Kingdom}

\section{Dynasty 18}

c. 1539-1292

Ahmose (Nebpehtire)

1539-1514

Amenophis I (Djeserkare)

1514-1493

Tuthmosis I (Akheperkare)

1493-1482

Tuthmosis II (Akheperenre)

1482-1479

Hatshepsut (Maatkare)

$1479-1458$

Tuthmosis III (Menkheperre)

$1479-1426$

Amenophis II (Akheprure)

1426-1400

Tuthmosis IV (Menkheprure)

1400-1390

Amenophis III (Nebmaatre)

1390-1353

Amenophis IV/Akhenaten (Neferkheprure)

$1353-1336$ 
Smenkhkare (Ankhkheprure)

1336-1333

Tutankhamun (Nebkheprure)

$1333-1323$

Aya (Kheperkheprure)

1323-1319

Haremhab (Djeserkheprure)

1319-1292

\section{Dynasty 19 (early Ramesside Period)}

c. $1292-1 \mid 88$

Ramesses I (Menpehtire)

1292-1290

Sethos I (Menmaatre)

1290-1279

Ramesses II (Usermaatre setepenre)

1279-1213

Merneptah (Baenre meriamun)

1213-1203

Sethos II (Userkheprure setepenre)

1203-1196

Amenmesse (Menmire)

Siptah (Akhenre setepenre)

1196-1190

Twosre (Sitre meritamun)

1190-1188

\section{Dynasty 20 (late Ramesside Period)}

c. | | 88-1075

Sethnakhte (Userkhaure meryamun)

Ramesses III (Usermaatre meryamun)

$1188-1186$

1186-1155

Ramesses IV (Heqamaatre setepenamun)

$1155-1148$

Ramesses V (Usermaatre sekheperenre)

$1148-1143$

Ramesses VI (Nebmaatre meryamun)

$1143-1135$

Ramesses VII (Usermaatre setepenre meryamun)

1135-1129

Ramesses VIII (Usermaatre akhenamun)

1129-1127

Ramesses IX (Neferkare setepenre)

1127-1108

Ramesses X (Khepermaatre setepenre)

1108-1104

Ramesses XI (Menmaatre setepenptah)

1104-1075

\section{Third Intermediate Period}

\section{Dynasty 21}

c. $1075-945$

Smendes (Hedjkheperre setepenre)

Amenemnisu (Neferkare)

Psusennes I (Akheperre setepenamun)

Amenemope (Usermaatre setepenamun)

Siamun (Netjerkheperre setepenamun)

Psusennes II (Titkheprure setepenre)

$$
\begin{array}{r}
1075-1044 \\
1044-1040 \\
1040-990 \\
993-984 \\
978-960 \\
960-945
\end{array}
$$

\section{Dynasty 22 (Bubastids)}

c. $945-715$

Shoshenq I (Hedjkheperre setepenre)

Osorkon I (Sekhemkheperre setepenre)

945-924

$924-889$ 
Takelot I (Hedjkheperre setepenre)

Osorkon II (Usermaatre setepenamun)

$889-874$

Takelot II (Hedjkheprre setepenre)

$874-850$

Shoshenq III (Usermaatre setepenre)

$850-825$

Pami (Usermaatre setepenre)

$825-773$

Shoshenq V (Akheperre setepenre)

$773-767$

$767-730$

Osorkon IV (Akheperre setepenamun)

730-715?

(additionally, many local principalities)

\section{Dynasty 23}

c. $818-715$

Pedubaste (Usermaatre setepenamun)

Shoshenq IV (Usermaatre meryamun)

818-793

Osorkon III (Usermaatre setepenamun)

$793-787$

$787-759$

Takelot III (Usermaatre setepenamun)

764-757

Rudjamun (Usermaatre setepenamun)

$757-754$

Iupet II (Usermaatre setepenamun)

(additionally, many local principalities)

754-715(?)

\section{Dynasty 24}

c. 725-7/2

Tefnakhte (Shepsesre)

$725-718$

Bocchoris (Wahkare)

$718-712$

\section{Dynasty 25 (Ethiopian)}

c. $712-664$

Kashta (Maatre ?)

?-740

Piye/Piankhy (?) (Usermaatre and others)

740-713

Shabaka (Neferkare)

$712-698$

Shebitku (Djedkaure)

$698-690$

Taharqa (Khunefertemre)

$690-664$

Tantamani (Bakare)

$664-656$

Assyrian Conquest

$671-664$

\section{Late Period}

\section{Dynasty 26 (Saite)}

$664-525$

Necho I (Menkheperre)

Psammetichus I (Wahibre)

672-664

664-610

Necho II (Wehemibre)

610-595

Psammetichus II (Neferibre)

595-589

Apries (Haaibre)

589-570 
Amasis (Khnemibre)

$570-526$

Psammetichus III (Ankhkaenre)

526-525

Dynasty 27 (Persian Period)

$525-404$

\section{Dynasty 28}

Amyrtaios

404-399

Dynasty 29

$399-380$

Nepherites I (Baenre merynetjeru)

399-393

Psammuthis (Userre setepenptah)

393

Hakoris (Khnemmaatre)

Nepherites II

393-380

380

Dynasty 30

$380-343$

Nectanebo (Nakhtnebef) I (Kheperkare)

Teos (Irmaatenre)

$380-362$

$362-360$

Nektanebo (Nakhthorehbit) II (Senedjemibre)

$360-343$

Second Persian Period

$343-332$

Macedonians

$332-305$

Ptolemaic Period

305-30

Roman and Byzantine Periods

30 B.C.E. -642 C.E. 


\section{H S T R Y - F \\ A N C I E N \\ E $⿴ 囗 十 \mathrm{P}$}

